\title{
PCR Typing of Mycobacterium ulcerans Lineages Based on Large Sequence Polymorphisms
}

Michael Kaser ${ }^{1,2 *}$, Julia Hauser ${ }^{1,2}$ and Gerd Pluschke ${ }^{1,2}$

${ }^{1}$ Swiss Tropical and Public Health Institute, Switzerland

${ }^{2}$ University of Basel, Switzerland

\begin{abstract}
Genetic fine typing of Mycobacterium ulcerans, the causative agent of the neglected tropical disease Buruli ulcer, has long been hampered by the remarkable genetic homogeneity of patient isolates. However, large sequence polymorphisms (LSPs), mainly originating from insertional-deletional genomic events, allow the differentiation between major lineages of $M$. ulcerans and other mycolactone producing mycobacteria (MPMs). Here, we describe an LSP-based rapid PCR typing scheme for these mycobacteria. Application of this approach to a selection of mycobacterial strains isolated from Buruli ulcer patients in Japan shows that they belong to the $M$. ulcerans asian haplotype.
\end{abstract}

Keywords: Genetic diversity; Large sequence polymorphisms; Bacterial phylogeny; Epidemiology; Buruli ulcer; Mycobacterium ulcerans

\section{Introduction}

Evolutionary affiliation of bacterial strains can be accomplished by various genetic typing methods [1]. Choice of the most suitable method depends on the population structure of the analysed species [2-4]. Large sequence polymorphisms (LSPs) are labour-intensive to identify in mycobacteria but constitute very reliable indicators for kinship and evolutionary order $[3,5,6]$. In particular, insertions/deletions (InDels) with identical sequence breakpoints, sequence signatures, and insertional gene content show unequivocal common ancestry and witness highly reliably bacterial phylogeny $[7-9,6,10]$.

Analysis of LSPs has enabled the genetic differentiation of major lineages in a world-wide collection of M. ulcerans strains [11,8]. Since M. ulcerans is undergoing reductive evolution [12] it has been suspected that ongoing genome reduction would allow the performance of genetic fine typing of local M. ulcerans populations for micro-epidemiological studies. However, a lack of further diversification of African M. ulcerans isolates was observed [13]. Still, in a worldwide collection of isolates, LSP analyses revealed an intra-species evolutionary scenario with two distinct phylogenetic lineages. M. ulcerans haplotypes from Asia, South America, and Mexico belong to the ancestral lineage, whereas the classical lineage includes haplotypes from Africa, Australia, and South East Asia. Altogether seven haplotypes based on LSPs have been described $[8,9]$ and a panel of LSPs has been identified to characterize the Asian M. ulcerans InDel haplotype $[8,14]$. Furthermore, studies including diverse "mycolactone producing mycobacteria" (MPMs) elucidated strain relatedness by InDel analysis within well defined regions of difference (RDs) [7].

M. ulcerans is the cause of Buruli ulcer, a chronic necrotizing skin disease, with a focus in West-Africa and Australia, but is found in tropical regions throughout the world [15-17]. In Japan and China sporadic cases of clinical Buruli ulcer (like) disease were reported. These were associated with pathogens that were given the (sub-) species names M. shinshuense or M. ulcerans ssp. Shinshuense [18-25]. Sequence analysis of the $16 \mathrm{~S}$ rRNA already suspected a close genetic relationship to M. Ulcerans $[26,25]$ albeit the position of $M$. shinshuense among pathogenic mycobacteria remained unclear.
The acquisition of the virulence plasmid pMUM001, encoding enzymes required for the synthesis of the cytotoxic polyketide mycolactone, is a key factor in the pathogenesis of Buruli ulcer [2729]. Compared to Buruli ulcer endemic areas of West Africa and Australia, where the classical lineage is found, the disease occurs only very sporadically in endemic regions where the ancestral lineage is distributed $[30,14,31]$. Genomic studies imply that strains belonging to the classical lineage have undergone patho-adaptive loss of genes $[29,12,27,4,14,32]$. Yet, it remains unclear at which point of evolution, in which geographic region, and by which selective pressure(s) enhanced virulence has emerged.

Here, we present a rapid PCR-based genotyping scheme for allocation of new $M$. ulcerans isolates into the previously defined InDel haplotypes. This analysis is easy to perform since it is based on amplification products (amplicons) obtained by conventional PCR designed for haplotype specific LSPs. As an example for the application of the method we have characterized a selection of Japanese mycobacterial strains associated with Buruli ulcer (like) clinical lesions, that have been given different (sub-)species names. Also, application of LSP analysis to RD6, which is highly variable between the lineages, indicates closer relatedness of the M. ulcerans classical lineage to the South American InDel haplotype than to other members of the ancestral lineage.

\section{Materials and Methods}

\section{Mycobacterial strains}

Strains used in this study are: $M$. marinum M (ATCC BAA-535) and ATCC927, origin of both strains as described in [33]; M. ulcerans

*Corresponding author: Michael Kaser, Swiss Tropical and Public Health Institute, Socinstr. 57, 4002Basel, Switzerland, Tel: +41-61-2848-365; Fax: +4161-2848-101; E-mail: m.kaeser@unibas.ch

Received December 08, 2011; Accepted January 17, 2012; Published January 19, 2012

Citation: Kaser M, Hauser J, Pluschke G (2012) PCR Typing of Mycobacterium ulcerans Lineages Based on Large Sequence Polymorphisms . J Medical Microbio Diagnosis S1:001. doi:10.4172/2161-0703.S1-001

Copyright: (c) 2012 Kaser M, et al. This is an open-access article distributed unde the terms of the Creative Commons Attribution License, which permits unrestricted use, distribution, and reproduction in any medium, provided the original author and source are credited. 
Ghana IFIK 1066089 (own collection); Malaysia ITM 941328, Australia ITM 9549, Australia ITM 5142, China ITM 980912, Japan 882/ITM 8756 [18] (ATCC 33728), Surinam ITM 842, origin of ITM strains as described in [11]; M. shinshuense 753 [21], strain kindly provided by P. Small, University of Tennessee, USA.

\section{Genomic DNA extraction}

DNA was extracted as described earlier [34,35]. Bacterial pellets of $20 \mathrm{mg}$ (wet weight) were heat inactivated for 1 hour at $95^{\circ} \mathrm{C}$ in $500 \mu \mathrm{l}$ PBS, resuspended in lysis buffer ( $15 \%$ sucrose, $0.05 \mathrm{M}$ Tris ( $\mathrm{pH} 8.5$ ), $0.05 \mathrm{M}$ EDTA), and sequentially treated with lysozyme $(10 \mathrm{mg} / \mathrm{ml}$, $\left.1 \mathrm{hr}, 37^{\circ} \mathrm{C}\right)$ and $\operatorname{SDS}(4 \%)$ and proteinase $\mathrm{K}(0.2 \mathrm{M}$ proteinase $\mathrm{K})(1$ $\mathrm{hr}, 37^{\circ} \mathrm{C}$ ). After enzyme deactivation and adding of $0.1 \mathrm{~mm}$ diameter zirconia beads, samples were homogenized using the Precellys 24 homogenizer (Bertin Technologies, Montigny-le-Bretonneux, France) at conditions of $3 \times 30 \mathrm{~s}$ at $6800 \mathrm{rpm}$. Supernatants were subjected to phenol-chloroform extraction and ethanol precipitation, and DNA concentration was determined using the NanoDrop Spectrophotometer ND-1000 (NanoDrop, Wilmington, DE) and adjusted to retrieve equal starting material.

\section{DNA amplification}

PCR was performed using FirePol 10x BD buffer and $0.5 \mu \mathrm{l}$ FirePolTaq-Polymerase (Solis BioDyne, Tartu, Estonia), 5 ng genomic DNA or the corresponding volume of nuclease free water as a negative control, $0.6 \mu \mathrm{M}$ forward and reverse primers each, $1.7 \mathrm{mM} \mathrm{MgCl}_{2}$ and $0.3 \mathrm{mM}$ of each dNTP in a total volume of $30 \mu \mathrm{l}$. Long-range PCR polymerase mix (Fermentas, St. Leon-Rot, Germany) was applied according to the manufacturer's protocol to retrieve PCR products longer than $3 \mathrm{~kb}$. PCR reactions were run in a GeneAmp PCR System 9700 PCR machine. The thermal profile for PCR amplification of mycobacterial genomic DNA included an initial denaturation step of $95-98^{\circ} \mathrm{C}$ for $3 \mathrm{~min}$, followed by $32 \mathrm{cycles}$ of $95^{\circ} \mathrm{C}$ for $20 \mathrm{sec}$, annealing at $58-65^{\circ} \mathrm{C}$ for $20 \mathrm{sec}$, and elongation at $72^{\circ} \mathrm{C}$ for $30 \mathrm{sec}$ upto $4 \mathrm{~min}$. The PCR reactions were finalized by an extension step at $72^{\circ} \mathrm{C}$ for $10 \mathrm{~min}$. PCR products were analyzed on $1 \%$ agarose gels by gel electrophoresis using ethidium bromide staining and the AlphaImager illuminator (Alpha Innotech, San Leandro, CA, USA). Primers (Sigma-Aldrich, Steinheim, Germany) were selected on the genome sequences of $M$. ulcerans Agy99 (Genbank accession number CP000325) and $M$. marinum M (Genbank accession number CP000854 and CP000895) using the Primer3 software (http://frodo.wi.mit.edu/cgi-bin/primer3/ primer3_www.cgi).

\section{Results and Discussion}

\section{LSP based haplotyping}

LSPs differentiating between $M$. ulcerans and M. marinum lineages were used to design primer pairs that allow easy and reliable amplification of haplotype-specific DNA sequences (Figure 1). In the case of RD12, such an amplicon (369 bp) clearly distinguishes the patient isolate M. marinum $\mathrm{M}$ from other mycobacteria due to the presence of a sequence feature (insertion InsRD12A containing the gene M_MAR3972) in that particular strain (Fig 1A,) [34]. Other $M$. marinum strains and MPMs yield a PCR product with different size (315 bp) in a duplex PCR reaction due to the absence of that particular insertion (Figure 1A). Using a set of selected (monoplex or duplex) PCR reactions targeting sequences in earlier defined RDs, PCR products specific for the established LSP haplotypes allow the positioning of new mycobacterial strains into the current genealogy (Figure 1B and C). With this amplicon typing scheme, all M. ulcerans haplotypes of the ancestral lineage can be determined, the two major lineages can be distinguished from each other, and two haplotypes can be assigned among the Australian M. ulcerans strains (Figure 1B and C).

\section{Affiliation of M. shinshuense}

We applied the above described PCR scheme to type a panel of Asian mycobacterial isolates. Mycobacterial strains from Asia (Japan, China, Malaysia) were tested for the presence of specific LSPs within six different RDs featuring unique characteristics for the Asian InDel haplotype (Figure 2). These InDel regions were earlier identified and characterized using isolates from Japan (ITM 8756) and China (ITM 980912) [8,14]. DNA from the Malaysian M. ulcerans strain showed features of both the classical and the ancestral lineage in the investigated RDs but may have an additional specific genetic signature in RD11. As expected [36,25], isolates of Japanese origin, irrespective of their (sub-) species designations, showed in all RDs genetic traits of the common M. ulcerans Asian InDel haplotype (Figure 2).

\section{Relationship between South American isolates and the classical lineage}

RD6 is a complex region with regard to LSP based genetic variations and therefore particularly suitable to differentiate between lineages. Gene segments from three different regions of an $M$. marinum-like genome were shuffled together in the $M$. ulcerans ancestral lineage, thereby inverting one segment (a $1 \mathrm{~kb}$ piece comprising M_MUL0504/0505) and forming the sequence situation found in the Asian haplotype in RD6 (Figure 2). Within this shuffled region, the South American haplotype shows a subsequent insertion of $21 \mathrm{~kb}$ through lateral gene transfer (containing 21 coding sequences of "insertion sequences and phages", M_MUL0508 through M_MUL0529, with 57-83\% identity over between 600-1900 bps, revealed by nucelotide fasta blasts, to genes of Actinobacteria other than $M$. marinum). This insertion left genetic marks at the edges that testify the genome shuffling in the order of evolutionary events from the M. marinum sequence via the Asian to the South American InDel haplotype sequences. Interestingly, this very sequence situation of the South American haplotype is shared by the $M$. ulcerans classical lineage which only shows yet additional copies of the insertion sequence elements (ISEs) IS2404 and IS2606 within this $21 \mathrm{~kb}$ insertion stretch. Hence, LSP analysis in this RD provides evidence for the classical lineage to show closer genetic kinship to the South American haplotype than to any other member of the ancestral lineage.

\section{Conclusion}

In this study, we demonstrate that a simple amplicon typing scheme based on InDels allows the distinction between major lineages of $M$. ulcerans. For instance, patient isolates from Japan that have been given different (sub-)species names, exhibited identical traits characteristic for the Asian M. ulcerans lineage. This supports the recent suggestion to give all MPMs, including M. shinshuense, the species name $M$. Ulcerans [36].

At some point in the M. ulcerans evolution, the mycolactone producing ancestor of the classical lineage has been equipped with particular virulence, most likely through ISE-associated genomic changes and at least in part through loss of highly immunogenic proteins $[12,14,32]$. The strength of LSP analyses to show unequivocal details of genetic signatures is very helpful in the development of 
Citation: Kaser M, Hauser J, Pluschke G (2012) PCR Typing of Mycobacterium ulcerans Lineages Based on Large Sequence Polymorphisms . J Medical Microbiol Diagnosis S1:001. doi:10.4172/2161-0703.S1-001

A

PCR No. 1 - schematic view of primer binding regions
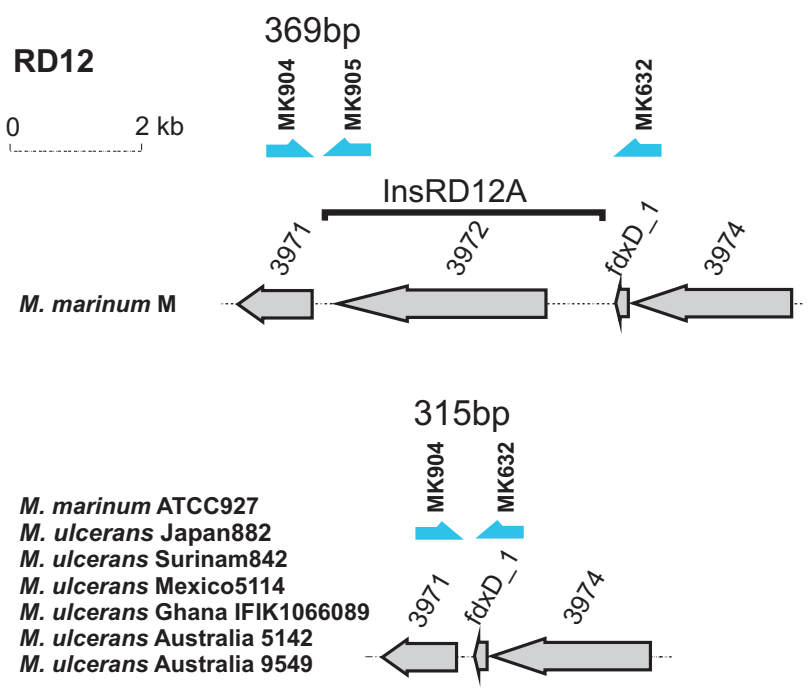

M. marinum ATCC927

M. ulcerans Japan882

M. ulcerans Surinam842

M. ulcerans Mexico5114

M. ulcerans Ghana IFIK1066089

M. ulcerans Australia 5142

M. ulcerans Australia 9549

PCR No. 1 - duplex PCR results

MK904 / MK905 / MK632

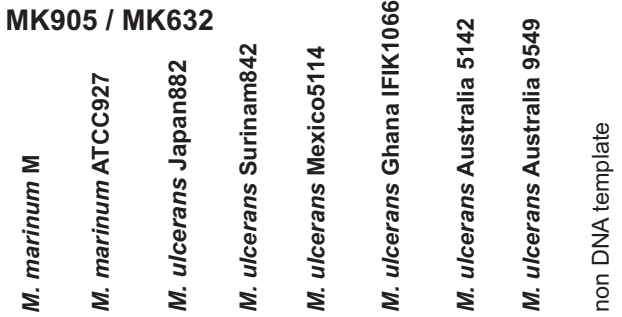

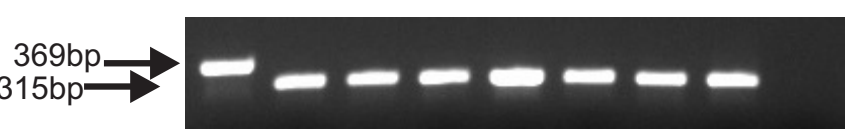

B Amplicon typing scheme flowchart

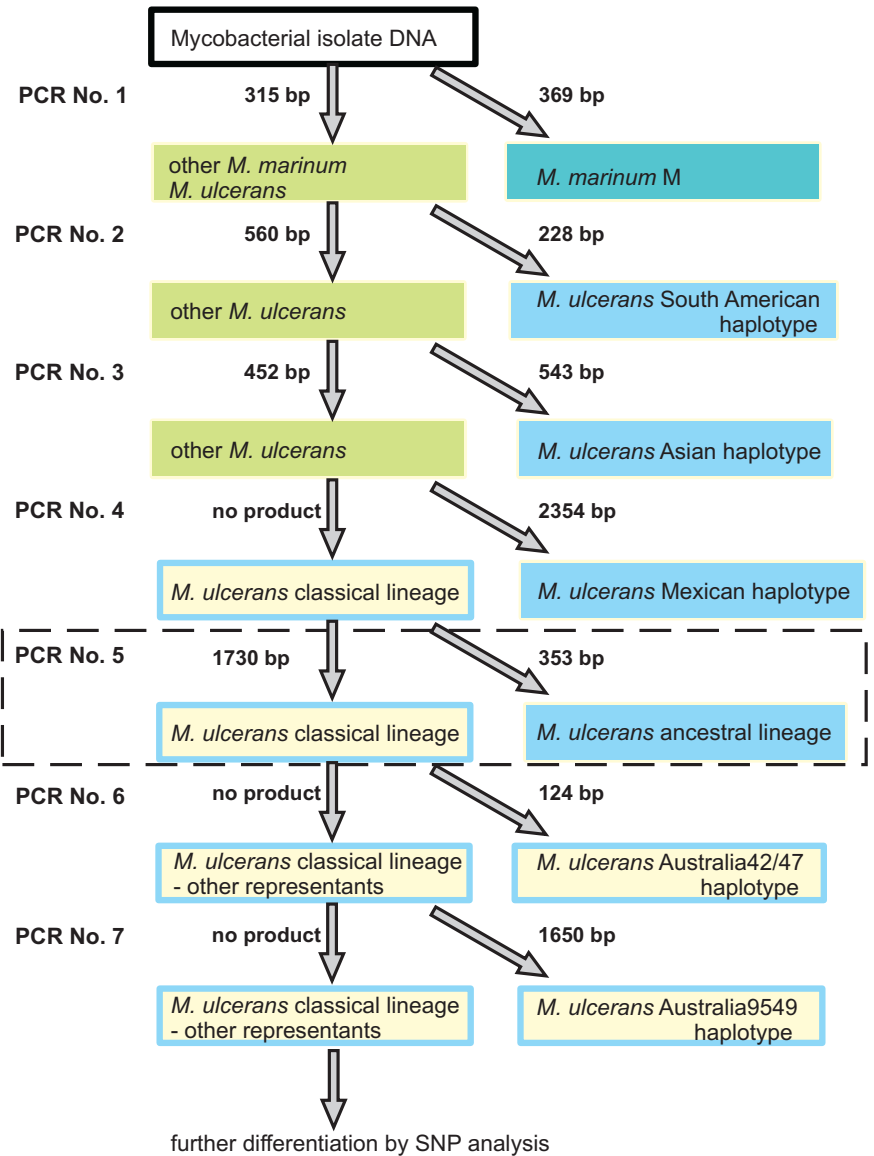

C Haplotype specific PCRs

\begin{tabular}{|c|c|c|c|c|c|c|}
\hline $\begin{array}{l}\text { PCR } \\
\text { No. }\end{array}$ & $\begin{array}{l}\text { Species/ lineage/ } \\
\text { InDel haplotype }\end{array}$ & Representing strain & RD & $\begin{array}{l}\text { Deletion/ } \\
\text { insertion }\end{array}$ & $\begin{array}{l}\text { confirming PCR product } \\
\text { size (bp) (primers) }\end{array}$ & $\begin{array}{l}\text { excluding PCR product } \\
\text { size (bp) (primers) }\end{array}$ \\
\hline 1 & M. marinum M & M. marinum $\mathrm{M}$ & 12 & InsRD12A & 369 (MK904/MK905) & 315 (MK904/MK632) \\
\hline 2 & $\begin{array}{l}\text { M. ulcerans South } \\
\text { American haplotype }\end{array}$ & M. ulcerans Surinam 842 & 12 & $\triangle \mathrm{RD} 12 \mathrm{~B}$ & 228 (MK328/MK689) & 560 (MK328/MK750) \\
\hline 3 & $\begin{array}{l}\text { M. ulcerans Asian } \\
\text { haplotype }\end{array}$ & M. ulcerans Japan 882 & 12 & $\triangle \mathrm{RD} 12 \mathrm{C}$ & 543 (MK907/MK629) & 452 (MK376/MK629) \\
\hline 4 & $\begin{array}{l}\text { M. ulcerans Mexican } \\
\text { haplotype }\end{array}$ & M. ulcerans Mexico 5114 & 13 & $\triangle \mathrm{RD} 13 \mathrm{~A}$ & $2354(\mathrm{CH} 18 / \mathrm{CH} 19)$ & na \\
\hline 5 & ancestral lineage & e.g. M. ulcerans Surinam 842 & 1 & no insertion & 353 (MK808/MK809) & na \\
\hline $\mathrm{J}$ & classical lineage & M. ulcerans Ghana IFIK1066089 & 1 & InsRD1(IS2404) & 1730 (MK808/MK809) & na \\
\hline 6 & $\begin{array}{l}\text { M. ulcerans } \\
\text { Australia42/47 haplotype }\end{array}$ & M. ulcerans Australia 5142 & 3 & $\triangle \mathrm{RD} 3 \mathrm{~A}$ & 124 MK205/MK204 & na \\
\hline 7 & $\begin{array}{l}\text { M. ulcerans Australia9549 } \\
\text { haplotype }\end{array}$ & M. ulcerans Australia 9549 & 13 & $\triangle R D 13 B$ & $1650(\mathrm{CH} 10 / \mathrm{CH} 11)$ & na \\
\hline
\end{tabular}

Figure 1: LSP based PCR typing scheme. (A): Schematic view of primer binding regions exemplified for PCR No. 1 in RD 12 and corresponding results of the duplex PCR for a panel of mycobacteria. In this example, M. marinum M is distinguished from all other analysed strains. (B): Amplicon typing scheme flowchart. Assay numbers and PCR product sizes correspond to the information given in table C. (C): Table of selected haplotype specific PCRs including the corresponding sets of primers. "na": not applicable. Primer sequence information is to be found in supplementary table S1. 
Citation: Kaser M, Hauser J, Pluschke G (2012) PCR Typing of Mycobacterium ulcerans Lineages Based on Large Sequence Polymorphisms . J Medical Microbiol Diagnosis S1:001. doi:10.4172/2161-0703.S1-001

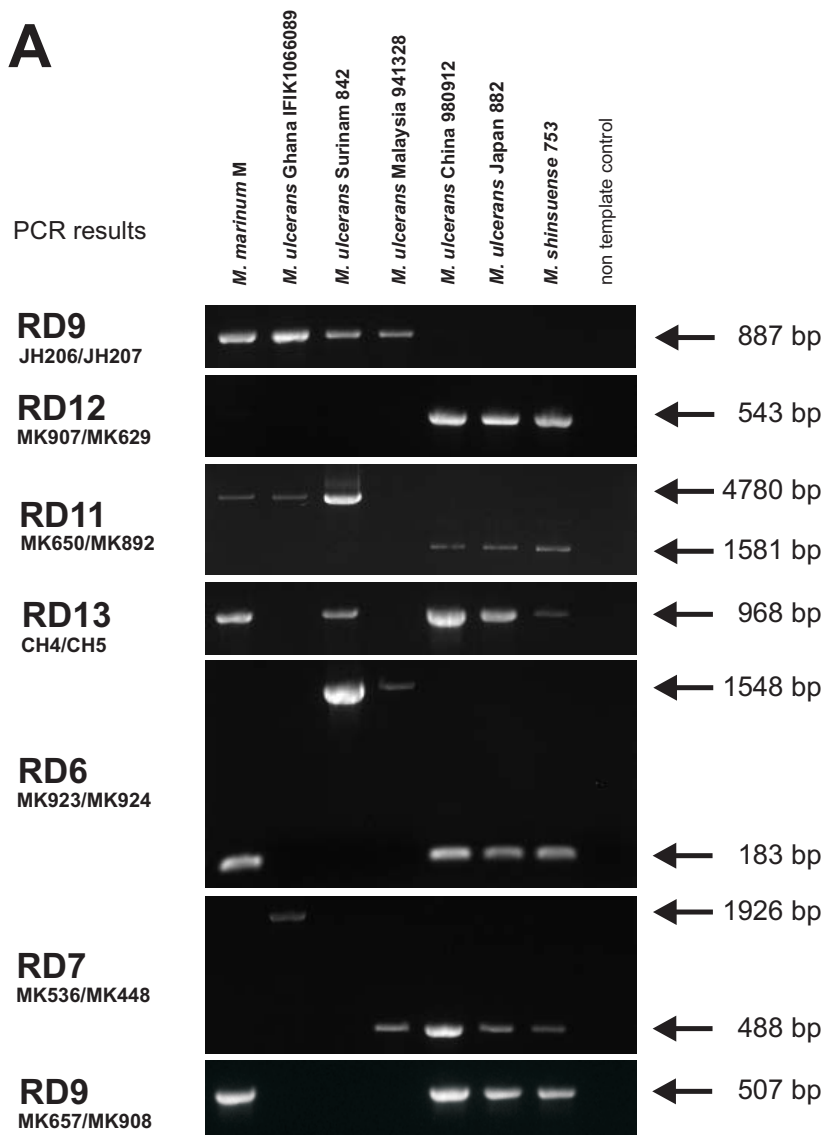

Figure 2: LSP based PCR typing of Asian strains. The Japanese strains $M$. ulcerans/M. shinshuense Japan 882 and $M$. shinshuense 753 were analysed together with M. ulcerans strains from China (ITM 980912) and Malaysia (ITM 941328), and compared with control strains of the South American haplotype (Surinam 842), a representative of the classical lineage (M. ulcerans Ghana IFIK1066089), and the patient isolate M. marinum M. Amplicons shown are (from top to down): RD9: 887 bp product covering M_MAR3536 which is deleted in $\triangle R D 9 A$ of the Asian InDel haplotype; RD12: $54 \overline{3}$ bp product bridging the large Asian deletion $\triangle \mathrm{RD} 12 \mathrm{C}$; RD11: 1581 bp product bridging the Asian deletion $\triangle R D 11 A$ ::IS2404 - the 4780 bp product reveals sequence preservation (M_MAR2557 through M_MAR2563) in other strains - strain Malaysia 941328 may have a specific genetic signature here; RD13: 968 bp product spanning genes esxB/esxA missing in the classical lineage; RD6: 183 bp product present in M. marinum and the M. ulcerans Asian InDel haplotype, but absent from the classical lineage - the 1548 bp product contains an IS2404 element carried by the M. ulcerans South American haplotype; RD7: 488 bp product specific for M. ulcerans but absent in M. marinum - a 1926 bp product is found only in the M. ulcerans classical lineage (contains a copy of IS2606), the South American haplotype carries here a secondary deletion; RD9: 507 bp product (within M MAR3547) only present in M. marinum and the M. ulcerans Asian InDel haplotype. For positions of the RDs, see [14]. Equal amounts of DNA were applied to the DNA amplification reactions. Primer sequence information is provided in supplementary table $\mathrm{S} 1$.

evolutionary scenarios. Application of next generation genome sequencing technologies will allow the refinement of these scenarios and to further elucidate the relationship between different lineages of M. ulcerans and other MPMs. While LSP analyses are not suitable to fine-type local M. ulcerans populations [13], whole genome SNP typing greatly enables microepidemiological studies to address open questions concerning the transmission of M. ulcerans $[12,13,37,38]$.

\section{Acknowledgements}

This research activity was supported by the "Stop Buruli"-initiative funded by the UBS Optimus Foundation. We thank Sara Stupan for technical assistance in very initial stages of the project.

\section{References}

1. Baker S, Hanage WP, Holt KE ( 2010) Navigating the future of bacterial molecular epidemiology. Curr Opin Microbiol 13: 640-645.

2. Brosch R, Pym AS, Gordon SV, Cole ST (2001) The evolution of mycobacterial pathogenicity: clues from comparative genomics. Trends Microbiol 9: 452-458.

3. Hirsh AE, Tsolaki AG, DeRiemer K, Feldman MW, Small PM (2004) Stable association between strains of Mycobacterium tuberculosis and their human host populations. Proc Natl Acad Sci USA 101: 4871-4876.

4. Yip MJ, Porter JL, Fyfe JA, Lavender CA, Portaels F, et al. (2007) Evolution of Mycobacterium ulcerans and other mycolactone-producing mycobacteria from a common Mycobacterium marinum progenitor. J Bacteriol 189: 2021-2029.

5. Gagneux S, DeRiemer K, Van T, Kato-Maeda M, de Jong BC, et al. (2006) Variable host-pathogen compatibility in Mycobacterium tuberculosis. Proc Nat Acad Sci USA 103: 2869-2873.

6. Alland D, Lacher DW, Hazbon MH, Motiwala AS, Qi W, et al. (2007) Role of large sequence polymorphisms (LSPs) in generating genomic diversity among clinical isolates of Mycobacterium tuberculosis and the utility of LSPs in phylogenetic analysis. J Clin Microbiol 45: 39-46.

7. Kaser M, Gutmann O, Hauser J, Stinear T, Cole S, et al. (2009). Lack of insertional-deletional polymorphism in a collection of Mycobacterium ulcerans isolates from Ghanaian Buruli ulcer patients. J Clin Microbiol 47: 3640-3646.

8. Kaser M, Rondini S, Naegeli M, Stinear T, Portaels F, et al. (2007) Evolution of two distinct phylogenetic lineages of the emerging human pathogen Mycobacterium ulcerans. BMC Evol Biol 7: 177.

9. Huber CA, Ruf MT, Pluschke G, Kaser M (2008) Independent loss of immunogenic proteins in Mycobacterium ulcerans suggests immune evasion. Clin Vaccine Immunol 15: 598-606.

10. Fleischmann RD, Alland D, Eisen JA, Carpenter L, White O, et al. (2002) Wholegenome comparison of Mycobacterium tuberculosis clinical and laboratory strains. J Bacteriol 184: 5479-5490.

11. Rondini S, Kaser M, Stinear T, Tessier M, Mangold C, et al. (2007) Ongoing genome reduction in Mycobacterium ulcerans. Emerg Infect Dis 13: 1008-1015.

12. Stinear TP, Seemann T, Pidot S, Frigui W, Reysset G, et al. (2007) Reductive evolution and niche adaptation inferred from the genome of Mycobacterium ulcerans, the causative agent of Buruli ulcer. Genome Res 17: 192-200.

13. Kaser M, Hauser J, Small P, Pluschke G (2009) Large sequence polymorphisms unveil the phylogenetic relationship of environmental and pathogenic mycobacteria related to Mycobacterium ulcerans. Appl Environ Microbiol 75 5667-5675.

14. Kaser M, Pluschke G (2008) Differential Gene Repertoire in Mycobacterium ulcerans Identifies Candidate Genes for Patho-Adaptation. PLoS Negl Trop Dis 2: e353.

15. WHO (2008) Buruli ulcer: progress report, 2004-2008, Weekly epidemiological record. World Health Organization Geneva 83: 145-156.

16. Sizaire V, Nackers F, Comte E, Portaels F (2006) Mycobacterium ulcerans infection: control, diagnosis, and treatment. Lancet Infect Dis 6: 288-296.

17. Wansbrough-Jones M, Phillips R (2006) Buruli ulcer: emerging from obscurity Lancet 367: 1849-1858.

18. Tsukamura, M, Mikoshiba $H$ (1982) A new Mycobacterium which caused skin infection. Microbiol Immunol 26: 951-955.

19. Tsukamura M, Kaneda K, Imaeda T, Mikoshiba H (1989) A taxonomic study on a mycobacterium which caused a skin ulcer in a Japanese girl and resembled Mycobacterium ulcerans. Kekkaku 64: 691-697.

20. Faber WR, Arias-Bouda LM, Zeegelaar JE, Kolk AH, Fonteyne PA, et al. (2000) First reported case of Mycobacterium ulcerans infection in a patient from China. Trans R Soc Trop Med Hyg 94: 277-279.

21. Kazumi Y, Ohtomo K, Takahashi M, Mitarai S, Sugawara I, et al. (2004) 
Citation: Kaser M, Hauser J, Pluschke G (2012) PCR Typing of Mycobacterium ulcerans Lineages Based on Large Sequence Polymorphisms . J Medical Microbiol Diagnosis S1:001. doi:10.4172/2161-0703.S1-001

Mycobacterium shinshuense isolated from cutaneous ulcer lesion of right lower extremity in a 37-year-old woman. Kekkaku 79: 437-441.

22. Nakanaga K, Ishii N, Suzuki K, Tanigawa K, Goto M, et al. (2007) Mycobacterium ulcerans subsp. shinshuense' isolated from a skin ulcer lesion: identification based on 16S rRNA gene sequencing. J Clin Microbiol 45: 3840-3843.

23. Funakoshi T, Kazumi Y, Okada R, Nishimoto K, Saito M, et al. (2009) Intractable ulcer caused by Mycobacterium shinshuense: successful identification of mycobacterium strain by $16 \mathrm{~S}$ ribosomal RNA 3'-end sequencing. Clin Exp Dermatol 34: e712-e715.

24. Kondo M, Kurokawa I, Ito Y, Yamanaka K, Yamazaki T, et al. (2009) Leg ulcer caused by Mycobacterium ulcerans ssp. shinshuense infection. Int J Dermatol 48: $1330-1333$

25. Nakanaga K, Hoshino Y, Yotsu RR, Makino M, Ishii N (2011) Nineteen Cases of Buruli Ulcer Diagnosed in Japan from 1980 to 2010. J Clin Microbiol 49: $3829-3836$.

26. Portaels F, Fonteyene PA, de Beenhouwer H, de Rijk P, Guedenon A, et al. (1996) Variability in 3' end of 16S rRNA sequence of Mycobacterium ulcerans is related to geographic origin of isolates. J Clin Microbiol 34: 962-965.

27. Stinear TP, Mve-Obiang A, Small PL, Frigui W, Pryor MJ, et al. (2004) Giant plasmid-encoded polyketide synthases produce the macrolide toxin of Mycobacterium ulcerans. Proc Natl Acad Sci USA 101: 1345-1349.

28. Stinear TP, Pryor MJ, Porter JL, Cole ST (2005) Functional analysis and annotation of the virulence plasmid pMUM001 from Mycobacterium ulcerans. Microbiology 151: 683-692.

29. Stinear TP, Seemann T, Harrison PF, Jenkin GA, Davies JK, et al. (2008) Insights from the complete genome sequence of Mycobacterium marinum on the evolution of Mycobacterium tuberculosis. Genome Res 18: 729-741.
30. Guerr H, Palomino JC, Falconi E, Bravo F, Donaires N, etal. (2008) Mycobacterium ulcerans Disease, Peru. Emerg Infect Dis 14: 373-377.

31. Mve-Obiang A, Lee RE, Portaels F, Small PL (2003) Heterogeneity of mycolactones produced by clinical isolates of Mycobacterium ulcerans: implications for virulence. Infect Immun 71: 774-783.

32. Demangel C, Stinear TP, Cole ST (2009) Buruli ulcer: reductive evolution enhances pathogenicity of Mycobacterium ulcerans. Nat Rev Microbiol 7: 5060.

33. Kaser M, Ruf MT, Hauser J, Marsollier L, Pluschke G (2009) Optimized method for preparation of DNA from pathogenic and environmental mycobacteria. Appl Environ Microbiol 75: 414-418.

34. Kaser M, Hauser J, Pluschke G (2009) Single nucleotide polymorphisms on the road to strain differentiation in Mycobacterium ulcerans. J Clin Microbio 47: 3647-3652.

35. Kaser M, Ruf MT, Hauser J, Pluschke G (2010) Optimized DNA Preparation from Mycobacteria. Cold Spring Harb Protoc : pdb.prot5408.

36. Pidot SJ, Asiedu K, Kaser M, Fyfe JA, Stinear TP (2010) Mycobacterium ulcerans and Other Mycolactone-Producing Mycobacteria Should Be Considered a Single Species. PLoS Negl Trop Dis 4: e663.

37. Roltgen K, Qi W, Ruf MT, Mensah-Quainoo E, Pidot SJ, et al. (2010) Single nucleotide polymorphism typing of Mycobacterium ulcerans reveals focal transmission of buruli ulcer in a highly endemic region of Ghana. PLoS Negl Trop Dis 4: e751.

38. Qi W, Kaser M, Roltgen K, Yeboah-Manu D, Pluschke G (2009) Genomic diversity and evolution of Mycobacterium ulcerans revealed by next-generation sequencing. PLoS Pathog 5: e1000580.

This article was originally published in a special issue, Kidney Diseases \& Treatment handled by Editor(s). Dr. Qian Qi, Mayo Clinic College of Medicine, United States 